\title{
Exhaled breath biopsy: a new cancer detection paradigm
}

\author{
Balkees Abderrahman*,1 \\ ${ }^{1}$ Department of Breast Medical Oncology, The University of Texas MD Anderson Cancer Center, 1155 Pressler, Unit 1354, Houston, \\ TX 77030, USA \\ *Author for correspondence: Tel.: +001 281435 0303; bhabderrahman@mdanderson.org
}
"Breath biopsy has the potential to serve as a triage or referral tool for patients with early-stage cancer, who are asymptomatic or display nonspecific symptoms."

First draft submitted: 19 February 2019; Accepted for publication: 11 March 2019; Published online: 12 April 2019

Keywords: breath analysis $\bullet$ breath biopsy • cancer detection • early cancer detection • FAIMS • mass spectrometry - PAN trial • VOCs analysis • VOCs measurement • volatile organic compounds

A pioneering breathalyzer has been designed to collect exhaled breath samples with biomarker volatile organic compounds (VOCs), which could be associated with inflammatory diseases, infectious diseases, cancer and potentially certain types of cancer [1-5]. After collection, samples are analyzed through heat desorbtion, gas chromatography and field asymmetric ion mobility spectrometry (FAIMS) next-generation detection microchip technology for established biomarker VOCs or mass spectrometry (MS) for biomarker VOC discovery. These sophisticated collection and analysis techniques forge Owlstone Medical Breath Biopsy ${ }^{\circledR}$ platform [6]. This relatively small-in-size breathalyzer is seeking to achieve a monumental mission; saving 100,000 lives and US $\$ 1.5$ billion in healthcare costs.

\section{Background}

Volatile organic compounds are carbon-containing compounds that are volatile enough to be detectable in the gas phase at room temperature. Vapor pressure lower than $0.1 \mathrm{mmHg}$ is a discriminant for volatility according to the US Environmental Protection Agency [7]. Such VOCs can be generated from altered cellular metabolism in many diseases and cancers [8-11], and can be measured through different technologies [12].

Marc van der Schee, Head of Clinical, at Owlstone Medical, describes VOCs in the terms "a wealth of valuable information about the health of an individual. Over 1000 VOCs in breath have already been identified" [13].

Measurements of VOCs have been used to assess counterterrorism, environmental contamination, and the flavor and fragrance industry. Analysis of exhaled VOCs has been in used in clinical practice in the forms of carbon 13 urea breath testing for Helicobacter pylori, exhaled nitric oxide (NO) in asthma, hydrogen-methane testing for small-bowel bacteria overgrowth and ethanol detection [14-17].

The field of VOCs detection and analysis is decorated with several Nobel Laureates. British chemist Francis Aston received the 1922 Nobel Prize in Chemistry for the invention of MS [18]. British biochemist Richard Synge and British chemist Archer Martin jointly received the 1952 Nobel Prize in Chemistry for the invention of partition chromatography [18]. American chemist Linus Pauling received two undivided Nobel Prizes (1954 in Chemistry and 1962 a Nobel Peace Prize for opposing weapons of mass destruction), and used gas chromatography to quantify 250 volatiles in a breath sample [19].

\section{A global priority}

In 2017, WHO, released 'Guide to cancer early diagnosis' [20], ahead of World Cancer Day (4 February); outlining three key steps to save lives through early cancer detection: enhance public awareness of different cancer symptoms and persuade the lay public to seek care when symptoms arise, invest in equipping the health services and training

Future $\because$ Medicine 
health personnel to conduct accurate and timely diagnostics, and ensure cancer patients can access safe and effective treatments without financial hardship.

Low- and middle-income countries are at further disadvantage of succeeding in implementing these steps; given the limited access to and availability of cancer detection, diagnostic and treatment tools.

\section{Financial \& health implications}

In 2014, cancer treatments cost US patients nearly US $\$ 4$ billion out of pocket [21]. Moreover, such cancer treatments occupied a significant proportion of the total US healthcare spending bill; with costs mounting to nearly US $\$ 87.8$ billion, spent on cancer-related healthcare [21].

From 2010 through 2015, cancer services cost the NHS roughly $£ 5$ billion annually, but if the cost to society as a whole - including costs related to loss of productivity - are taken into consideration, as they should be, the costs inflate to nearly $£ 18.3$ billion annually [22]. In 2011, the UK government published 'Improving outcomes: a strategy for cancer' [23]; investing a budget of $£ 750$ million over 5 years to ensure early cancer diagnosis, and facilitate access to cancer testing and treatment.

Early cancer detection, evidently, remains a health and financial priority worldwide. Early and lower cost cancer detection is the first major area Owlstone Medical's Breath Biopsy (BB) platform is tapping into and is vowing to benefit. ReCIVA ${ }^{\circledR}$ Breath Sampler is noninvasive, pain free, has a relatively lower cost compared with other cancer detection methods, takes 1 min to collect exhaled breath with known biomarker VOCs or 10 min for biomarker VOC discovery, is a reproducible conduit for breath collection, and can be used anywhere not just the hospital. The BB cartridge captures every VOC form exhaled breath and does not require special handling upon shipping. Owlstone's FAIMS technology provides rapid, sensitive and selective VOC analysis. Such analysis can be conducted in a central lab or - on the long term - at point of care.

In the USA, costs pertaining to the management of adverse drug reactions (ADRs) go up to nearly US $\$ 30.1$ billion annually [24]. In the UK, definitely avoidable ADRs cost the NHS roughly £98.5 million annually; occupying 181,626 bed days and contributing to 1708 deaths during the index hospitalization [25]. These high costs are attributed to increased hospitalization (primary care ADRs), or prolongation of hospital stay and the need for more clinical investigations in serious cases (secondary care ADRs).

The ADRs, evidently, remain a health and financial dilemma worldwide. Avoiding ADRs upon drug administration - or precision drug administration - with a lower cost approach is the second major area Owlstone Medical's BB technology is tapping into and is vowing to benefit.

Chris Claxton, Head of Investor Relations, at Owlstone Medical, shared with us that exogenous VOCs (EVOCs) can be used to assess in vivo metabolic function using EVOC probes, which are comprised of generally recognized as safe compounds that are metabolized by the same enzyme system (CYP450) as drugs are. Claxton expanded that through measuring the kinetics of a mix of EVOC probe substrates and matabolites, a metabolizer phenotype can be generated. Changes in metabolizer phenotype can lead to drug toxicity or inefficacy. This is currently under development and will be launched initially for research applications.

\section{Landmark trial}

Owlstone Medical launched the PAN Cancer trial for Early Detection of Cancer in Breath (ClinicalTrials.gov Identifier: NCT03756597) [5] in collaboration with researchers from Cancer Research UK (London, UK), and the University of Cambridge and Cambridge University Hospitals NHS Foundation Trust (Cambridge, UK). This 1500-patient, six-cancer-targeted trial, is recruiting patients at Addenbrooke's Hospital in Cambridge with suspected oesophageal and gastric cancers. Over time, prostate, renal, bladder, liver and pancreatic cancer patients will be recruited. These cancer patients will represent the trial's cases, whereas recruited healthy individuals will represent their matched controls for gender, age and tumor-specific risk factors.

The trial's primary outcome is to detect cancer biomarker VOCs, which are linked to the early stages of these six cancers and discriminate biomarker VOCs between these six cancers if possible. Secondary outcomes include patients' feedback on ReCIVA's ${ }^{\circledR}$ usability and accessibility. Other outcomes include the influence of the tumor's primary location and its phenotype on VOC profiling.

Thrilling as this technology may be, several questions remain in store: will this breathalyzer be affected by other confounding variables such as the difference in patient characteristics (i.e., obese vs normal weight patients, or patients taking certain drugs affecting their metabolism vs those who are not)? Will the difference in patient treatment modalities which subsequently affect metabolism for the same cancer, produce divergent signature VOC 
profiles (i.e., chemotherapy vs immunotherapy for colon cancer)? And will the difference in patients' respective environments play a role?

Rebecca Fitzgerald at Cambridge University, lead PAN cancer trial investigator, spoke to us on these issues, and elaborated: "almost certainly (the answer to your first question), which is why we are including a large number of controls, both those being referred for a suspected cancer, but who turn out not to have cancer and are completely healthy volunteers." "We are collecting the samples prior to therapy, answering your second question." "We are sampling fairly large numbers of cases and controls and also trying to standardize the collections (such as using the) same consulting room, (at) random time of the day (not the same time for controls and different time for cases, etc), answering your third question."

\section{Triage \& cancer recurrence monitoring}

Breath biopsy has the potential to serve as a triage or referral tool for patients with early-stage cancer, who are asymptomatic or display nonspecific symptoms. Patients can be referred from primary care into specialized care. Specialists can, subsequently, implement more advanced testing (e.g., tissue biopsy) to further investigate their disease.

In addition, VOCs measurement and analysis has the potential to serve as an effective cancer recurrence monitoring tool. Markar and colleagues [26] conducted a study, whereby the results of the VOCs in breath, were shown to be a biomarker of colorectal cancer recurrence after surgery.

\section{The way forward}

Standardization of breath collection and analysis is a first vital step to widely incorporate the breath test into clinical practice. Breath tests can be influenced by methodology of breath collection, test environment and patients' physiologic condition. In 2005, the American Thoracic Society and European Respiratory Society published international recommendations for standardizing NO measurement [27]. This spurred its adoption as a diagnostic tool.

Standardizing the instruments used for the exhaled breath test represents a second vital step. Such instruments should be tested and optimized to become reliable in reproducing the VOCs results.

Separating patients with different tumor subtypes, tumor stages, histologic and molecular subtypes, from controls according to established test thresholds is a third vital step before instigating masked validations.

Accurate clinical reporting based on established guidelines (e.g., Standards for Reporting of Diagnostic Accuracy Studies [STARD] [28] and CONSORT [29]) is a fourth vital step.

Establishing large, multicenter clinical studies with appropriate patient populations, and external validations against positive controls, is a fifth vital step.

\section{Financial \& competing interests disclosure}

This work was supported by the NIH, MD Anderson's Cancer Center support grant number: CA016672 (to P Pisters), the George and Barbara Bush Foundation for Innovative Cancer Research (to VC Jordan, OBE, FMedSci) and the Dallas/Fort Worth Living Legend Fellowship of Cancer Research (to B Abderrahman). B Abderrahman would like to thank her mentor VC Jordan for his support. The author has no other relevant affiliations or financial involvement with any organization or entity with a financial interest in or financial conflict with the subject matter or materials discussed in the manuscript apart from those disclosed.

No writing assistance was utilized in the production of this manuscript.

\section{References}

1. Arasaradnam RP, McFarlane MJ, Ryan-Fisher C et al. Detection of colorectal cancer (CRC) by urinary volatile organic compound analysis. PLoS ONE 9(9), e108750 (2014).

2. Owlstone Medical. LuCID: a multi-centre prospective trial for lung cancer screening (2019). www.owlstonemedical.com/clinical-pipeline/lucid/

3. Owlstone Medical. InTERCEPT: the early detection of colorectal cancer aiming to save thousands of lives every year (2019). www.owlstonemedical.com/clinical-pipeline/intercept/

4. Owlstone Medical. STRATA: selecting the right treatment for severe asthma patients by replacing the trial and error approach (2019). www.owlstonemedical.com/clinical-pipeline/strata/

5. Clinical Trials database: NCT03756597. https://clinicaltrials.gov/ct2/show/NCT03756597 
6. Owlstone Medical Ltd. Owlstone Medical's Breath Biopsy ${ }^{\circledR}$ Platform (2019). www.owlstonemedical.com/science-technology/breath-biopsy/

7. Environmental Protection Agency. Definition of volatile organic compound. (1978). www3.epa.gov/ttn/naaqs/aqmguide/collection/Doc_0016_VOC330701781.pdf

8. De Lacy Costello B, Amann A, Al-Kateb H et al. A review of the volatiles from the healthy human body. J. Breath. Res. 8(1), 014001 (2014).

9. Gaude E, Frezza C. Tissue-specific and convergent metabolic transformation of cancer correlates with metastatic potential and patient survival. Nat. Commun. 7, 13041 (2016).

10. Warburg O. On the origin of cancer cells. Science 123(3191), 309-314 (1956).

11. Tennant DA, Duran RV, Gottlieb E. Targeting metabolic transformation for cancer therapy. Nat. Rev. Cancer 10(4), 267-277 (2010).

12. Queralto N, Berliner AN, Goldsmith B, Martino R, Rhodes P, Lim SH. Detecting cancer by breath volatile organic compound analysis: a review of array-based sensors. J. Breath. Res. 8(2), 027112 (2014).

13. Van Der Schee M, Pinheiro H, Gaude E. Breath biopsy for early detection and precision medicine in cancer. Ecancermedicalscience 12, ed84 (2018).

14. Harding P, Field PH. Breathalyzer accuracy in actual law enforcement practice: a comparison of blood- and breath-alcohol results in Wisconsin drivers. J. Forensic Sci. 32(5), 1235-1240 (1987).

15. Atreja A, Fu AZ, Sanaka MR, Vargo JJ. Non-invasive testing for Helicobacter pylori in patients hospitalized with peptic ulcer hemorrhage: a cost-effectiveness analysis. Dig. Dis. Sci. 55(5), 1356-1363 (2010).

16. Petrone P, Sarkisyan G, Fernandez M et al. Small intestinal bacterial overgrowth in patients with lower gastrointestinal symptoms and a history of previous abdominal surgery. Arch. Surg. 146(4), 444-447 (2011).

17. Robroeks CM, Van Berkel JJ, Jobsis Q et al. Exhaled volatile organic compounds predict exacerbations of childhood asthma in a 1-year prospective study. Eur. Respir. J. 42(1), 98-106 (2013).

18. Hübschmann H-J. Chapter 3: The historical development of the GC-MS technique. In: Handbook of GC-MS: Fundamentals and Applications (3rd Edition). Wiley-VCH, Weinheim, Germany. (2015).

19. Pauling L, Robinson AB, Teranishi R, Cary P. Quantitative analysis of urine vapor and breath by gas-liquid partition chromatography. Proc. Natl Acad. Sci. USA 68(10), 2374-2376 (1971).

20. WHO. Guide to cancer early diagnosis. (2017). https://apps.who.int/iris/bitstream/handle/10665/254500/9789241511940-eng.pdf;js essionid=4BEC54447715A45A8F035BCC110C119C?sequence $=1$

21. Cancer Action Network American Cancer Society. The costs of cancer: addressing patient costs. (2017). www.fightcancer.org/sites/default/files/Costs\%20of\%20Cancer\%20-\%20Final\%20Web.pdf

22. UK Government Department of of Health and Social Care. 2010 to 2015 government policy: cancer research and treatment. (2015). www.gov.uk/government/publications/2010-to-2015-government-policy-cancer-research-and-treatment/2010-to-2015-governmentpolicy-cancer-research-and-treatment

23. UK Government Department of of Health and Social Care. Improving outcomes: a strategy for cancer. (2011). www.gov.uk/government/publications/the-national-cancer-strategy

24. Sultana J, Cutroneo P, Trifiro G. Clinical and economic burden of adverse drug reactions. J. Pharmacol. Pharmacother. 4(Suppl. 1), S73-S77 (2013).

25. Policy Research Unit in Economic Evaluation of Health \& Care Interventions (EEPRU). Prevalence and economic burden of medication errors in the NHS in England (2018) www.eepru.org.uk/wp-content/uploads/2018/02/eepru-report-medication-error-feb-2018.pdf 29- www.consort-statement.org/

26. Markar SR, Chin ST, Romano A et al. Breath volatile organic compound profiling of colorectal cancer using selected ion flow-tube mass spectrometry. Ann. Surg. 269(5), 903-910 (2019).

27. American Thoracic Society, European Respiratory Society. ATS/ERS recommendations for standardized procedures for the online and offline measurement of exhaled lower respiratory nitric oxide and nasal nitric oxide, 2005. Am. J. Respir. Crit. Care Med. 171(8), 912-930 (2005).

28. Cohen JF, Korevaar DA, Altman DG et al. STARD 2015 guidelines for reporting diagnostic accuracy studies: explanation and elaboration. BMJ Open 6(11), e012799 (2016).

29. Consort Transparent Reporting of Trials. CONSORT 2010 statement: updated guidelines for reporting parallel group randomised trials (2010). 